\title{
Gause's exclusion principle revisited: artificial modified species and competition
}

\author{
J. C. Flores ${ }^{\dagger}$ and R. Beltran ${ }^{\ddagger}$ \\ †Universidad de Tarapacá, Departamento de Física, Casilla \\ 7-D, Arica, Chile. \\ łUniversidad de Tarapacá, Departamento de Matemáticas, \\ Casilla 7-D, Arica, Chile.
}

\begin{abstract}
Gause's principle of competition between two species is studied when one of them is sterile. We study the condition for total extinction in the niche, namely, when the sterile population exterminates the native one by an optimal use of resources. A mathematical Lotka-Volterra non linear model of interaction between a native and sterile species is proposed. The condition for total extinction is related to the initial number $M_{o}$ of sterile individuals released in the niche. In fact, the existence of a critical sterile-population value $M_{c}$ is conjectured from numerical analysis and an analytical estimation is found. When spatial diffusion (migration) is considered a critical size territory is found and, for small territory, total extinction exist in any case. This work is motived by the extermination agriculture problem of fruit flies in our region.
\end{abstract}

Published in: Jour.Phys.A:Math.Gen. 33, 4877 (2000).

PACS:

87.23.C Population Dynamics (Ecology).

02.30 Nonlinear Differential Equations. 
In ecological systems Gause's exclusion principle is widely accepted [1-5]. Originally it was deducted from competition between Paramecium caudatum and Paramecium aurelia [1-3]. Nevertheless, it applies to many other situations. For instance, in reference [6] was conjectured that Neanderthal extinction in Europe was consequence of Gause's principle. In a formal point of view, it states that two competing species cannot coexist in the same ecological niche. In this framework, it is assumed that the strong species fulls completely the niche and the weak disappears (exclusion). We remark that this principle is limited in the sense that it applies when re-adaptation, migration, or genetic changes does not exist. This principle seems very intuitive in natural environment or for species in laboratories; but what is the situation with genetically prepared sterile populations ?. To be more explicit, consider the well known problem related to extermination of native fruit flies by genetically sterilized fruit flies $[7,8]$. The two species exist in the same ecological niche when the sterile population is released. Before the interaction, we assume that the native species fulls the niche in a stable way. In some geographic regions and for optimal initial conditions, native fruit flies can be exterminated by the sterile population. Namely, in this case both species disappears and the principle must be reformulated as

Principle of Gause: Two competing species cannot coexist in the same ecological niche and at least one of the species disappear.

Namely, it contains explicitly the possibility of total extinction (both species). This formulation of the principle includes all strategy of extermination with genetically altered species $[7,8]$.

In this paper we consider a mathematical non linear model of competition between a native species with number of individuals $N(t)$, and other sterile 
with number of individuals given by $M(t)$. Explicitly, we are interested on the mathematical conditions for total extinction in the ecological niche. The structure of the article is the follows: first we present a predator-predator non linear model for the variable $N$ and $M$ (equations 1-3), and its stability analysis. Numerical simulation confirms the stability analysis and the existence of a threshold $M_{c}$ where total extinction exist. We give an analytical estimation of this threshold value (equation (5)). Near to the critical value, the behavior of the extinction time $\tau_{\text {ext }}$ is studied. This extinction time is, after our numerical calculation, related to an critical exponent $\nu$ (equation (6)). Finally, the case including diffusion is considered, here we found the existence of a critical size territory $L_{c}$ were total extinction holds (for any initial condition of the species $M$ ). Some possible generalizations are stressed at the end of the paper.

To be explicit, consider the Lotka-Volterra type evolution equations with interaction

$$
\begin{aligned}
& \frac{d M}{d t}=-\alpha^{\prime} M-\delta N M, \\
& \frac{d N}{d t}=N F(N)-\delta N M,
\end{aligned}
$$

where $\alpha^{\prime}$ is the death-rate of the sterile population, $\delta$ is the interaction parameter, and the function $F(N)$ describes the population growing of the native species when interaction does not exist. For instance, when $F(N)=\alpha-N$ ( $\alpha$ is a constant) we obtain the usual Verlhust, or logistic, equation. Remark that the stability of the point $(N=0, M=0)$ depends of the sign of $F(0)$. In fact, when $F(0)<0$ this point becomes stable and the possibility of total extinction exists in accordance with the Gause's principle. Moreover, this condition of stability of $(0,0)$ seems reasonable if we think that species needs a minimal social structure, or genetical diversity, to survival (i.e. a minimal 
number of individuals). We stress that the dynamical systems (1-3) is irreversible, for instance, a Lyapounov function $L$ associated to the systems is just $L=M(t)$.

To carry-out explicit our calculations we consider the model where

$$
F(N)=(\alpha-N)(N-\beta),
$$

with $\alpha$ and $\beta$ positives parameters $(\alpha<\beta)$. Since $F(0)<0$, the point $(N=0, M=0)$ is stable and total extinction would be expected. The linear analysis of (1-3) shows that the point $(N=\alpha, M=0)$ is unstable (saddle) and $(N=\beta, M=0)$ is a stable focus. Figure 1 shows the stability diagram for our equations. So, the sterile population $M$ disappears and, depending on the initial conditions, total extinction would exist in the niche. Namely, the systems has two atractors, the first $(0,0)$ related to total extinction and the other $(\beta, 0)$ related to survival of species $N$.

Figure 1: A sketch of the critical points associated with equations (1-2) 
The explicit question which we are concerned in this paper is the follows: if initially the native species number is $N=\beta$ (the stable point without interaction) then, after released $M_{o}$ sterile individuals, when have we total extinction?. Namely, before the interaction, the native species is in the niche in a stable way. After, $M_{o}$ sterile individuals are released and the interaction process produces a competitive struggle. Here we ask about the minimal population $M_{o}$ of sterile individuals producing total extinction in the niche. In fact, if the sterile population is not enough then they death and total extermination do not occur.

Numerical calculations confirm the existence of a critical value $M_{c}$ and when $M_{o}>M_{c}$ total extinction exist in the niche. Figure 2 shows the time behaviors of $N(t)$ for different initial value $M_{o}$, of the sterile species released in the niche. There is a critical value for the initial condition $M_{o}$ related to total extinction. A criterion for total extinction is depending on the initial number of sterile population $M_{o}$ and given by

$$
M_{o}>2.7 \frac{(\alpha-\beta)^{2}}{4 \delta} .
$$

This criterion is established as follows: from (1) and (3), we have that $M(t)=$ $\exp \left(-\alpha^{\prime}-\delta<N>_{t}\right) t$, where $<N>_{t}=(1 / t) \int{ }^{t} N(\tau) d \tau$. When $t \rightarrow \infty$, and assuming total extinction, we expect an exponential decaying behavior for $M$. So, an important fraction of the decaying process, assumed slow, is reached when $M \sim M_{o} e^{-1}$. If now we stressed that not other stationary point (excepting $(0,0)$ exist in $(2)$, we obtain the criterion (4). We have used the maximum value of the function $F(N)$ given by (3). We remark that this is a coarse criterion, nevertheless, it works in accord with our numerical simulations. For instance, the figure 2 describes extinction when $M_{0}>270$ 
in accordance with (4). This is true also for other parameter values.

Figure 2: A numerical simulations of equations (1-3)

The criteria (4) can be generalized easily to a system with arbitrary distribution $F(N)$ in (1-2). Namely, by imposing the inequality $F_{\max }<\delta M_{o} e^{-1}$ (with $e \sim 2,7$ ).

The figure 3 shows a simulation of the final native population $N(t=\infty)$ for different initial condition $M_{o}$ of the sterile population. Clearly there is a critical value $M_{c}$ which separates the survival and extermination regime. From equation (4), a first approximation for this critical values is

$$
M_{c} \sim 2.7 \frac{(\alpha-\beta)^{2}}{4 \delta} .
$$


Figure 3: The final distribution of native population $M$

Moreover, figure 2 suggests that near to this critical value the extinction time $\tau_{\text {ext }}$ depends on $\left(M_{o}-M_{c}\right)$. This is so because when $M_{o} \rightarrow M_{c}^{+}$the extinction time must be infinity. Explicitly we expect a behavior like

$$
\tau_{e x t} \sim \frac{1}{\left(M_{o}-M_{c}\right)^{\nu}} ; M_{o}>M_{c},
$$

where $\nu$ is a unknown parameter. The evaluation of this critical exponent requires a computational work beyond of the scope of this paper. It will be do elsewhere. The conjecture (6) is reinforced by numerical calculation. In fact, using the same parameter values that in figure 2 , and the definition

$$
\tau_{\text {ext }}^{-1}=-\frac{1}{t} \lim _{t \rightarrow \infty} \operatorname{Ln}(N(t) / \beta),
$$

we see that the existence of the critical exponent $\nu$ is confirmed numerically (figure 4). 
Figure 4: The extinction time $\tau_{\text {ext }}$

Now we discuss briefly the incorporation of migration to the set of evolution equations (1-2). In fact, total extermination could be also carried out by diffusion process. In some cases unstable points become stable by diffusion in limited territories. Thus in a model where $(0,0)$ is unstable, i.e. only one species survive, diffusion would change this instability and total extinction takes place. We add the diffusion terms $D_{M} \frac{\partial^{2} M}{\partial x^{2}}$ and $D_{N} \frac{\partial^{2} N}{\partial x^{2}}$ to (1) and (2) respectively. Namely, consider the pair of reaction-diffusion evolution equations

$$
\begin{aligned}
& \frac{d M}{d t}=-\alpha^{\prime} M-\delta N M+D_{M} \frac{\partial^{2} M}{\partial x^{2}}, \\
& \frac{d N}{d t}=N F(N)-\delta N M+D_{N} \frac{\partial^{2} N}{\partial x^{2}} .
\end{aligned}
$$

The linear analysis of stability for the stationary point $(0,0)$ can be do in 
the usual way, namely, consider the small perturbation

$$
\begin{aligned}
& M=0+\eta, \\
& N=0+\varepsilon,
\end{aligned}
$$

where the variables $\eta$ and $\varepsilon$ are assumed small. Equations (8) and (9), give the first order linear equations

$$
\begin{gathered}
\frac{\partial \eta}{\partial t}=-\alpha^{\prime} M \eta+D_{M} \frac{\partial^{2} \eta}{\partial^{2} x} \\
\frac{\partial \varepsilon}{\partial t}=F(0) \varepsilon+D_{N} \frac{\partial^{2} \varepsilon}{\partial^{2} x}
\end{gathered}
$$

where we assume $F(0)>0$, corresponding to the unstable case when migration is not present. For finite territory, solutions like $\varepsilon \sim e^{\omega t} \sin k x$ can be visualized. The relationship between the stability parameter $\omega$ and the wavenumber $k$ is given by

$$
\omega=F(0)-k^{2} D_{N}
$$

and clearly for $k>\sqrt{F(0) / D_{N}}$ the point $(0,0)$ becomes stable and total extinction in the niche exist. Since $k \sim \frac{1}{L}$, with $L$ the territory size, equation (14) defines a critical size territory $L_{c} \sim \sqrt{D_{N} / F(0)}$ where total extinction holds. Namely, for all size territory $L<L_{c}$ total extinction exist.

In conclusion: the principle of Gause was generalized to consider the case of biological struggle when one competing species is sterile. In fact, under appropriate conditions, total extinction could occur in the niche. Most of the agriculture competitive extermination method are carried-out assuming this principle. For instance, this is the case of extermination program of fruit flies with sterile flies irradiated in laboratories [9-10]. We have presented a simple model which has total extinction in the niche in some cases. Conjectures 
related to a critical sterile-population (number of individuals) producing total extinction were pointed-out with a coarse criterion (4). This conjectures are based in our numerical simulation of the model. The role of migration was briefly discussed and the possibility of total extinction from diffusion was also explored for small territories.

To ending, we note that our model can be extended to incorporates some modifications. Particularly we are thinking about generalizations like:

(i) Periodic variation of coefficients. In fact, in the extinction fruit flies programs, daily, season or El niño (ENSO), oscillations must be considered.

(ii) Sexual selection. Many extermination programs are based on sexual selection, namely, sterile-male released in a given niche. It creates interaction between sterile-male and fertile-female which becomes directly related to the evolution of the native-male. Such models require a phase-space greater than two.

(iii) Many random factors are present in real niche. For instance, humidity, temperature, wind, etc. These factors can be incorporated in our model by introducing adequate sthocastic process for the parameter $(\alpha, \beta$, or $\delta)$.

Acknowledgment: we thanks Hernan Donoso (S. A. G. Arica) for introduce us at the problem of extinction of fruit flies with sterile populations. We thanks C. Romo for the revision of the manuscript. J. C. F. thanks E. Martin and C. Saravia for the initial help concerning the subject.

\section{References}

[1] Gause G F (1934) The Struggle For Existence. Willians and Willians, Baltimore . 
[2] Emmel T C (1973) Ecology and Population Biology. W. W. Norton, New York .

[3] Villee C A (1977), Biology, W. B. Saunders Co.

[4] Wio H S, Kuperman M N, Von Haeften B, Bellini M, Deza R R and Schat C (1996) Instabilities and Nonequilibrium Structures (Tirapegui E and Zeller W, Editors). Kluwer

[5] Murray J D (1989) Mathematical Biology. Springer-Varlag.

[6] Flores J C (1998) J.Theor.Biol. 181, 295.

[7] Aluja M and Liedo P Ed. (1990), Fruit Flies : Biology and Management. Springer-Verlag .

[8] A S Robinson and Hooper G, Ed. (1989) In: Fruit Flies, Their Biology, Natural Enemies and Control. Amsterdam, Elsevier.

[9] McInnis D O , Tam S, Grace C and Miyashida P (1994) Ann.Entomol.Soc.Am. 87, 231.

[10] Vargas R I, Miyashita D, Nishida T (1984). Ann.Entomol.Soc.Am. 77, 651. 